\title{
FRACTIONAL VARIATIONAL PROBLEMS ON CONFORMABLE
} CALCULUS

\author{
Süleyman ÖĞREKÇİ and Serkan ASLIYÜCE \\ Department of Mathematics, Amasya University, 05100 Amasya, TURKEY
}

\begin{abstract}
In this paper, we deal with the variational problems defined by an integral that include fractional conformable derivative. We obtained the optimality results for variational problems with fixed end-point boundary conditions and variable end-point boundary conditions. Then, we studied on the variational problems with integral constraints and holonomic constraints, respectively.
\end{abstract}

\section{INTRODUCTION}

Origin of fractional calculus dates back to 1600's, firstly seen in a letter from Leibnitz to L'Hospital. So far, a number of famous mathematicians such as Abel, Fourier, Liouville, Leibnitz, Weyl and Riemann made contributions to this theory. Probably, Abel has given the first applications of fractional calculus in 1823. Especially in last decades, fractional calculus find ample applications in various fields of science (see 13, 22, 23, 27, 28]). Recently, fractional order Black-Scholes equation is studied in 11], fractional Harry-Dym equation is studied in [12]. There are several definitions of fractional derivatives and fractional integrals, such as Atangana-Baleanu, Riemann-Liouville, Grunwald-Letnikov, Caputo, Riesz, RieszCaputo, Hadamard-Hilfer, Caputo-Fabrizio, and Weyl, etc. We refer to monographs 15, 20, 24 for definitions and properties of most common fractional derivatives. Recently Khalil et. al. 19] gave a new well-behaved fractional derivative definition; named as conformable fractional derivative. This new definition has many similar properties with ordinary integer order derivative such as constant function rule, linearity, product and quotient rules and Leibnitz rule (see [1]). Conformable fractional differential equations are studied widely in the literature. We refer to 9

2020 Mathematics Subject Classification. 49K30, 26A33.

Keywords and phrases. Conformable fractional derivative, calculus of variations, subsidiary conditions.

suleyman.ogrekci@amasya.edu.tr; serkan.asliyuce@amasya.edu.tr-Corresponding author

(D) 0000-0003-1205-6848; 0000-0003-1729-3914.

(C) 2021 Ankara University Communications Faculty of Sciences University of Ankara-Series A1 Mathematics and Statistics 
for Lie symmetry analysis; to 8 for boundary value problems; to 18 for numerical solutions conformable differential equations; to 14 for Fourier transform, etc.

Calculus of variations is a subject which is concerned with finding the maxima and minima of functionals and plays important role in many problems arising in mechanics, geometry, analysis etc. We refer to monograph 17 for the basic concepts of this theory. In 1996, Riewe 25] noted that the traditional Lagrangian and Hamiltonian mechanics can not be used with non-conservative forces. In order to deal with Lagrangians involving nonconservative forces, Riewe 26] generalized the usual variational methods by using Riemann-Liouville type operators and introduced the fractional order calculus of variations. For different definitions on fractional derivatives, different approaches have been developed to generalize calculus of variations to fractional case. Agarwal 2, 3, 4, studied variational methods for Riemann-Liouville, Caputo and Riesz fractional derivatives. Almeida 5, 6 considered variational problems involving Riesz-Caputo and Caputo-Katugampola fractional derivatives. Zhang et. al. 29] and Bastos 7] studied calculus of variations with Caputo-Fabrizio derivatives. Chatibi et. al. 10] investigated variational methods for Atangana-Baleanu fractional derivatives. Lazo and Torres [21] and Eroğlu and Yapışkan 16 studied variational methods for conformable fractional derivatives.

In this paper, we consider more general variational problems with conformable fractional derivative and extend the results given in 21. More specially, we investigate variable end-point variational problems and variational problems with subsidiary conditions.

\section{Preliminaries}

In this section, we introduce definitions and basic properties concerning the conformable fractional derivative that will be needed in our proofs.

$0<\alpha \leq 1$ order left-conformable fractional derivative of the function $h$ : $[a, \infty) \rightarrow \mathbb{R}$ is defined by

$$
\left(T_{\alpha}^{a} h\right)(t):=\lim _{\varepsilon \rightarrow 0} \frac{h\left(t+\varepsilon(t-a)^{1-\alpha}\right)-h(t)}{\varepsilon} .
$$

If $\left(T_{\alpha} h\right)$ exists on the interval $(a, b)$, then $\left(T_{\alpha}^{a} h\right)(a)=\lim _{t \rightarrow a^{+}}\left(T_{\alpha}^{a} h\right)(t)$.

Similarly, $0<\alpha \leq 1$ order right-conformable fractional derivative of the function $h$ is defined by

$$
\left({ }_{\alpha}^{b} T h\right)(t):=\lim _{\varepsilon \rightarrow 0} \frac{h\left(t+\varepsilon(b-t)^{1-\alpha}\right)-h(t)}{\varepsilon} .
$$

If $\left({ }_{\alpha}^{b} T h\right)$ exists on the interval $(a, b)$, then $\left({ }_{\alpha}^{b} T h\right)(a)=\lim _{t \rightarrow b^{-}}\left({ }_{\alpha}^{b} T h\right)(t)$.

We remark that, additionally if $h$ is differentiable, then $\left(T_{\alpha}^{a} h\right)()=.(.-a)^{1-\alpha} h^{\prime}($. and $\left({ }_{\alpha}^{b} T h\right)()=.-(b-.)^{1-\alpha} f^{\prime}($.$) for all t \in(a, b)$. As in the case Caputo derivative, conformable derivative of the constant function is zero (see [1, 19]). 
$0<\alpha \leq 1$ order left and right conformable fractional integrals of the function $h$ are defined by

$$
\left(I_{\alpha}^{a} h\right)(t):=\int_{a}^{t} h(s) d^{\alpha}(s, a)
$$

and

$$
\left({ }_{\alpha}^{b} I h\right)(t):=\int_{t}^{b} h(s) d^{\alpha}(b, s)
$$

respectively, where $d^{\alpha}(s, a)=(s-a)^{\alpha-1} d s$ and $d^{\alpha}(b, s)=(b-s)^{\alpha-1} d s$ (see [1]).

Let $0<\alpha \leq 1$. If $h:[a, \infty) \rightarrow \mathbb{R}$ is continuous, then the identity $\left(T_{\alpha}^{a} I_{\alpha}^{a} h\right)(t)=$ $h(t)$ holds for all $t>a$. And, if $h:(a, \infty) \rightarrow \mathbb{R}$ is continuous, then the identity $\left(I_{\alpha}^{a} T_{\alpha}^{a} h\right)(t)=h(t)-h(a)$ holds for all $t>a($ see [1,19]).

For the differentiable functions $h, g:[a, b] \rightarrow \mathbb{R}$, the conformable integration by parts formula reads as follows (see [1])

$$
\int_{a}^{b} h(t)\left(T_{\alpha}^{a} g\right)(t) d^{\alpha}(t, a)=\left.(h g)(t)\right|_{t=a} ^{t=b}-\int_{a}^{b} g(t)\left(T_{\alpha}^{a} h\right)(t) d^{\alpha}(t, a) .
$$

In the following, we give the fundamental lemma of fractional variational calculus and the definition of jointly-convex functions that will be used in the sequel .

Lemma $1([21])$. Let the functions $\varphi, \xi:[a, b] \rightarrow \mathbb{R}$ be continuous and the the equality

$$
\int_{a}^{b} \varphi(t) \xi(t) d^{\alpha}(t, a)=0
$$

holds for all $\xi \in \mathcal{C}[a, b]$ satisfying $\xi(a)=\xi(b)=0$. Then

$$
\varphi(t)=0
$$

for all $t \in[a, b]$.

Definition 2 ( $[7])$. Let $F\left(x_{1}, x_{2}, x_{3}\right)$ be continuous function for its second and third arguments. If the inequality

$F\left(x_{1}, x_{2}+h_{1}, x_{3}+h_{2}\right)-F\left(x_{1}, x_{2}, x_{3}\right) \geq(\leq) \partial_{2} F\left(x_{1}, x_{2}, x_{3}\right) h_{1}+\partial_{3} F\left(x_{1}, x_{2}, x_{3}\right) h_{2}$

is hold for all $\left(x_{1}, x_{2}, x_{3}\right) \in A$ and all $h_{1}, h_{2} \in \mathbb{R}$, then we say that function $F$ is jointly-convex (or jointly-concave) in $A \subseteq \mathbb{R}^{3}$. 


\section{Main RESUlts}

In this study, we consider the functional

$$
\mathbb{J}[x]:=\int_{a}^{b} L\left(t, x(t), T_{\alpha}^{a} x(t)\right) d^{\alpha}(t, a) .
$$

Throughout the paper, we assume that $x \in \mathcal{C}^{1}[a, b], T_{\alpha}^{a}\left(\partial_{3} L\left(t, x(t), T_{\alpha}^{a} x(t)\right)\right)$ is continuous, and $L \in \mathcal{C}_{2,3}^{1}\left([a, b] \times \mathbb{R}^{2}, \mathbb{R}\right)$, where $\mathcal{C}_{2,3}^{1}$ denotes the family of functions that continuously differentiable for its second and third arguments, and $\partial_{i}$ denotes the partial derivative of the function for its $i$-th argument.

One can find necessary optimality conditions for the problem of finding local minimizers of the functional 2 in the following result.

Theorem $3\left([13)\right.$. Let $0<\alpha \leq 1$ and $x_{a}, x_{b} \in \mathbb{R}$ be fixed. If $x$ is a minimizer of the (2) on the set

$$
S:=\left\{x \in \mathcal{C}^{1}[a, b]: x(a)=x_{a}, x(b)=x_{b}\right\},
$$

then we say that $x(t)$ is a solution of the equation

$$
\partial_{2}\left(L_{\alpha, x}\right)-T_{\alpha}^{a}\left(\partial_{3}\left(L_{\alpha, x}\right)\right)=0
$$

where $L_{\alpha, x}:=L\left(., x(),. T_{\alpha}^{a} x().\right)$.

Definition 4. Equation (4) is named as the Euler-Lagrange equation for (2), and its solutions are named as the extremals of (2).

Equation (4) provides only a necessary condition for the function $x(t)$ to be an extremal of (2). By using notion of jointly-convex functions given above and conformable integration by parts formula (1), we can give a sufficient condition as follows.

Theorem 5. If the function $L$ is jointly-convex in $[a, b] \times \mathbb{R}^{2}$, then every solution of the Euler-Lagrange equation (4) minimizes the functional $\mathbb{J}$ on the set $S$.

Proof. Assume that function $x(t)$ is a solution of (4). Let $x+\epsilon \xi$ be a variation of $x$, with $1 \gg|\epsilon|$ and $\xi \in \mathcal{C}^{1}[a, b]$ with $\xi(a)=\xi(b)=0$. Since $x(t)$ is a solution of (4) and $L$ is jointly-convex, we have

$$
\begin{aligned}
& \mathbb{J}[x+\epsilon \xi]-\mathbb{J}[x] \\
= & \int_{a}^{b}\left(L_{\alpha, x+\epsilon \xi}\right) d^{\alpha}(t, a)-\int_{a}^{b}\left(L_{\alpha, x}\right) d^{\alpha}(t, a) \\
\geq & \epsilon \int_{a}^{b}\left[\partial_{2}\left(L_{\alpha, x}\right) \xi(t)+\partial_{3}\left(L_{\alpha, x}\right) T_{\alpha}^{a} \xi(t)\right] d^{\alpha}(t, a) .
\end{aligned}
$$




$$
=\epsilon \int_{a}^{b} \partial_{2}\left(L_{\alpha, x}\right) \xi(t) d^{\alpha}(t, a)+\epsilon \int_{a}^{b} \partial_{3}\left(L_{\alpha, x}\right) T_{\alpha}^{a} \xi(t) d^{\alpha}(t, a)
$$

Using (1) for the second term of the inequality, we can write

$$
\begin{aligned}
& \mathbb{J}[x+\epsilon \xi]-\mathbb{J}[x] \\
\geq & \epsilon \int_{a}^{b} \partial_{2}\left(L_{\alpha, x}\right) \xi(t) d^{\alpha}(t, a)+\left.\epsilon \xi(t) \partial_{3} \partial_{3}\left(L_{\alpha, x}\right)\right|_{t=a} ^{t=b} \\
& -\epsilon \int_{a}^{b} T_{\alpha}^{a}\left(\partial_{3}\left(L_{\alpha, x}\right)\right) \xi(t) d^{\alpha}(t, a) \\
= & \epsilon \int_{a}^{b}\left[\partial_{2}\left(L_{\alpha, x}\right)-T_{\alpha}^{a}\left(\partial_{3}\left(L_{\alpha, x}\right)\right)\right] \xi(t) d^{\alpha}(t, a) \\
= & 0 .
\end{aligned}
$$

Hence, we can say that $x(t)$ is a local minimizer of the functional $\mathbb{J}$.

In Theorems 3 and 5, we introduced the variational problems with fixed endpoint. In the following result, we will study the variational problems with variable end-point. Because of the absence of at least one of the end-point conditions (3) in such problems, we need additional conditions, which are named transversality conditions in the literature.

Theorem 6. Assume that $x(t)$ is a minimizer of the functional $\mathbb{J}$. Then, $x(t)$ is a solution of the Euler-Lagrange equation (4).

If $x(a)$ is absent, then

$$
\left.\partial_{3}\left(L_{\alpha, x}\right)\right|_{t=a}=0
$$

If $x(b)$ is absent, then

$$
\left.\partial_{3}\left(L_{\alpha, x}\right)\right|_{t=b}=0 .
$$

Proof. Let $x+\epsilon \xi$ be a variation of $x$, with $1 \gg|\epsilon|$ and $\xi \in \mathcal{C}^{1}[a, b]$. Let the functional $j$ defined in a neighborhood of zero by

$$
j[\epsilon]:=\mathbb{J}[x+\epsilon \xi] .
$$

Since $x$ is a minimizer of $\mathbb{J}$, then $\epsilon=0$ will be a minimizer of $j$ and so we can conclude that $j^{\prime}[0]=0$. Using [1], we can calculate $j^{\prime}[\epsilon]$ as

$$
\frac{\partial}{\partial \epsilon} j[\epsilon]=\frac{\partial}{\partial \epsilon}\left(\int_{a}^{b} L_{\alpha, x+\epsilon \xi} d^{\alpha}(t, a)\right)
$$




$$
\begin{aligned}
= & \int_{a}^{b}\left[\partial_{2}\left(L_{\alpha, x+\epsilon \xi}\right) \xi(t)+\partial_{3} L\left(L_{\alpha, x+\epsilon \xi}\right) T_{\alpha}^{a} \xi(t)\right] d^{\alpha}(t, a) \\
= & \int_{a}^{b} \partial_{2}\left(L_{\alpha, x+\epsilon \xi}\right) \xi(t) d^{\alpha}(t, a)+\left.\partial_{3}\left(L_{\alpha, x+\epsilon \xi}\right) \xi(t)\right|_{t=a} ^{t=b} \\
& -\int_{a}^{b} T_{a}^{\alpha}\left(\partial_{3}\left(L_{\alpha, x+\epsilon \xi}\right)\right) \xi(t) d^{\alpha}(t, a) .
\end{aligned}
$$

Using the fact that $j^{\prime}[0]=0$, we get

$$
\int_{a}^{b}\left[\partial_{2}\left(L_{\alpha, x}\right)-\partial_{3}\left(L_{\alpha, x}\right)\right] \xi(t) d^{\alpha}(t, a)+\left.\partial_{3}\left(L_{\alpha, x}\right) \xi(t)\right|_{t=a} ^{t=a}=0 .
$$

Also, since $x$ is a minimizer, the relation

$$
\partial_{2}\left(L_{\alpha, x}\right)-\partial_{3}\left(L_{\alpha, x}\right)=0
$$

holds for all $t \in[a, b]$. Therefore, from (5) we have

$$
\left.\partial_{3}\left(L_{\alpha, x}\right) \xi(t)\right|_{t=a} ^{t=a}=0 .
$$

If $x(a)$ is not fixed, then $\xi(a)$ will be free. Hence taking the end-point conditions as $\xi(a) \neq 0$ and $\xi(b)=0$, we obtain that

$$
\left.\partial_{3}\left(L_{\alpha, x}\right) \xi(t)\right|_{t=a}=0 .
$$

If $x(b)$ is not fixed, then $\xi(b)$ will be free. Hence taking the end-point conditions as $\xi(b) \neq 0$ and $\xi(a)=0$, we obtain that

$$
\left.\partial_{3}\left(L_{\alpha, x}\right) \xi(t)\right|_{t=b}=0 .
$$

Thus, the proof is complete.

Now we consider variational problems with constraints, i.e. subsidiary conditions. Let $l \in \mathbb{R}$ fixed, $G \in \mathcal{C}_{2,3}^{1}\left([a, b] \times \mathbb{R}^{2}, \mathbb{R}\right)$, and $T_{\alpha}^{a}\left(\partial_{3}\left(G_{\alpha, x}(t)\right)\right)$ is continuous.

Theorem 7. Assume that $x$ is a minimizer of functional (2), defined on the set (3) subject to the additional restriction

$$
\mathbb{I}[x]:=\int_{a}^{b} G_{\alpha, x} d^{\alpha}(t, a)=l .
$$

If $x$ is not an extremal of $\mathbb{I}$, then there exists a $\mu \in \mathbb{R}$ such that $x$ is a solution of the equation

$$
\partial_{2}\left(K_{\alpha, x}\right)-\partial_{3}\left(K_{\alpha, x}\right)=0
$$

where $K:[a, b] \times \mathbb{R}^{2} \rightarrow \mathbb{R}$ is defined by $K=L+\mu G$. 
Proof. Consider a variation of $x$ with two parameters $x+\epsilon_{1} \xi_{1}+\epsilon_{2} \xi_{2}$; with $1 \gg|\epsilon|$ and $\xi_{i} \in \mathcal{C}^{1}[a, b]$ satisfying $\xi_{i}(a)=\xi_{i}(b)=0$; for $i=1,2$. In the neighborhood of zero, let define the bivariate functions $k$ and $j^{*}$ as

$$
k\left(\epsilon_{1}, \epsilon_{2}\right)=\mathbb{I}\left(x+\epsilon_{1} \xi_{1}+\epsilon_{2} \xi_{2}\right)
$$

and

$$
j^{*}\left(\epsilon_{1}, \epsilon_{2}\right)=\mathbb{J}\left(x+\epsilon_{1} \xi_{1}+\epsilon_{2} \xi_{2}\right) .
$$

Using integration by parts formula given by (1), we obtain

$$
\begin{aligned}
& \frac{\partial}{\partial \epsilon_{2}} k\left(\epsilon_{1}, \epsilon_{2}\right) \\
& =\frac{\partial}{\partial \epsilon_{2}}\left(\int_{a}^{b} G_{\alpha, x+\epsilon_{1} \xi_{1}+\epsilon_{2} \xi_{2}} d^{\alpha}(t, a)-\int_{a}^{b} G_{\alpha, x} d^{\alpha}(t, a)\right) \\
& =\int_{a}^{b} \partial_{2}\left(G_{\alpha, x+\epsilon_{1} \xi_{1}+\epsilon_{2} \xi_{2}}\right) \xi_{2}(t) d^{\alpha}(t, a)+\int_{a}^{b} \partial_{3}\left(G_{\alpha, x+\epsilon_{1} \xi_{1}+\epsilon_{2} \xi_{2}}\right) T_{\alpha}^{a} \xi_{2}(t) d^{\alpha}(t, a) \\
& =\int_{a}^{b} \partial_{2}\left(G_{\alpha, x+\epsilon_{1} \xi_{1}+\epsilon_{2} \xi_{2}}\right) \xi_{2}(t) d^{\alpha}(t, a)+\left.\partial_{3}\left(G_{\alpha, x+\epsilon_{1} \xi_{1}+\epsilon_{2} \xi_{2}}\right) \xi_{2}(t)\right|_{t=a} ^{t=b} \\
& -\int_{a}^{b} T_{\alpha}^{a}\left[\partial_{3}\left(G_{\alpha, x+\epsilon_{1} \xi_{1}+\epsilon_{2} \xi_{2}}\right)\right] \xi_{2}(t) d^{\alpha}(t, a) \\
& =\int_{a}^{b}\left[\partial_{2}\left(G_{\alpha, x+\epsilon_{1} \xi_{1}+\epsilon_{2} \xi_{2}}\right)-T_{\alpha}^{a}\left[\partial_{3}\left(G_{\alpha, x+\epsilon_{1} \xi_{1}+\epsilon_{2} \xi_{2}}\right)\right]\right] \xi_{2}(t) d^{\alpha}(t, a) \\
& +\left.\partial_{3}\left(G_{\alpha, x+\epsilon_{1} \xi_{1}+\epsilon_{2} \xi_{2}}\right) \xi_{2}(t)\right|_{t=a} ^{t=b} .
\end{aligned}
$$

Therefore, we have

$$
\left.\frac{\partial}{\partial \epsilon_{2}} k\left(\epsilon_{1}, \epsilon_{2}\right)\right|_{(0,0)}=\int_{a}^{b}\left[\partial_{2}\left(G_{\alpha, x}\right)-T_{\alpha}^{a}\left[\partial_{3}\left(G_{\alpha, x}\right)\right]\right] \xi_{2}(t) d^{\alpha}(t, a)+\left.\partial_{3}\left(G_{\alpha, x}\right) \xi_{2}(t)\right|_{t=a} ^{t=b} .
$$

From the hypothesis we know that $x$ is not an extremal of $\mathbb{I}$, so we can conclude that there exists a function $\xi_{2}$ such that $\left.\frac{\partial}{\partial \epsilon_{2}} k\left(\epsilon_{1}, \epsilon_{2}\right)\right|_{(0,0)} \neq 0$. From the Implicit Function Theorem, we can say that there exists a unique function $\epsilon_{2}($.$) defined in$ the neighborhood of zero such that $k\left(\epsilon_{1}, \epsilon_{2}\left(\epsilon_{1}\right)\right)=0$ is satisfied.

Additionally, $(0,0)$ is a minimizer of $j^{*}$, with condition $k(.,)=$.0 , and so we proved that $\nabla k(0,0)=0$. After that using the Lagrange multiplier rule, we conclude that there exists a $\mu \in \mathbb{R}$ such that $\nabla(j+\mu k)=0$ is satisfied. Differentiating 
the map $\epsilon \rightarrow j\left(\epsilon_{1}, \epsilon_{2}\right)+\mu k\left(\epsilon_{1}, \epsilon_{2}\right)$, and taking $\left(\epsilon_{1}, \epsilon_{2}\right)=(0,0)$

$$
\int_{a}^{b}\left[\partial_{2}\left(G_{\alpha, x}\right)-T_{\alpha}^{a}\left[\partial_{3}\left(G_{\alpha, x}\right)\right]\right] \xi_{2}(t) d^{\alpha}(t, a)+\left.\partial_{3}\left(G_{\alpha, x}\right) \xi_{2}(t)\right|_{t=a} ^{t=b}=0
$$

is obtained. Finally, using the fundamental lemma, we obtain the desired result.

Now we consider variational problems with holonomic constraints, i.e. the case when admissible functions lie on a certain surface. Let the function $L \in$ $\mathcal{C}_{2,3,4,5}^{1}\left([a, b] \times \mathbb{R}^{4}, \mathbb{R}\right)$, and the functions $T_{\alpha}^{a}\left(\partial_{i} L\left(t, x_{1}(t), x_{2}(t), T_{\alpha}^{a} x_{1}(t), T_{\alpha}^{a} x_{2}(t)\right)\right)$ are continuous for $i=4,5$.

Consider the functional

$$
\mathbb{J}\left[x_{1}, x_{2}\right]:=\int_{a}^{b} L\left(t, x_{1}(t), x_{2}(t), T_{\alpha}^{a} x_{1}(t), T_{\alpha}^{a} x_{2}(t)\right) d^{\alpha}(t, a)
$$

on the space

$$
S^{*}:=\left\{\left(x_{1}, x_{2}\right): x_{1,2} \in \mathcal{C}^{1}[a, b],\left(x_{1}(a), x_{2}(a)\right)=x_{a} \text { and }\left(x_{1}(a), x_{2}(b)\right)=x_{b}\right\}
$$

where $x_{a}, x_{b} \in \mathbb{R}^{2}$ are fixed, and assume that the admissible functions of (8) lie on the surface

$$
G\left(t, x_{1}(t), x_{2}(t)\right)=0
$$

where $G \in \mathcal{C}_{2,3}^{1}\left([a, b] \times \mathbb{R}^{2}, \mathbb{R}\right)$.

For the sake of brevity, we denote $x(t):=\left(x_{1}(t), x_{2}(t)\right)$ and $T_{\alpha}^{a} x(t):=\left(T_{\alpha}^{a} x_{1}(t), T_{\alpha}^{a} x_{2}(t)\right)$ in the remaining part of this paper.

Theorem 8. Let $x \in S^{*}$ be a minimizer of $\mathbb{J}$ given by (8) under the constraint (9). If

$$
\partial_{3} G(t, x(t)) \neq 0, t \in[a, b],
$$

then there is a continuous function $\gamma:[a, b] \rightarrow \mathbb{R}$ such that $x$ satisfies

$$
\partial_{2}\left(L_{\alpha, x}\right)-T_{\alpha}^{a}\left(\partial_{4}\left(L_{\alpha, x}\right)\right)+\gamma(t) \partial_{2} G(t, x(t))=0,
$$

and

$$
\partial_{3}\left(L_{\alpha, x}\right)-T_{\alpha}^{a}\left(\partial_{5}\left(L_{\alpha, x}\right)\right)+\gamma(t) \partial_{3} G(t, x(t))=0 .
$$

Proof. Consider a variation of $x$ as $x+\epsilon \xi$ with $1 \gg|\epsilon|$ and $\xi \in \mathcal{C}^{1}[a, b]$ satisfying end-point conditions $\xi(a)=\xi(b)=0$. Since

$$
\partial_{3} G(t, x(t)) \neq 0,
$$

from the Implicit Function Theorem, we can say that there exists a subfamily of variations satisfying condition (9), i.e., there exists a unique function $\xi_{2}\left(\epsilon, \xi_{1}\right)$ such that $\left(x_{1}+\epsilon \xi_{1}, x_{2}+\epsilon \xi_{2}\right)$ satisfies (7). Therefore, we get

$$
G\left(t, x_{1}(t)+\epsilon \xi_{1}(t), x_{2}(t)+\epsilon \xi_{2}(t)\right)=0, t \in[a, b] .
$$


Differentiating equation (11) with respect to $\epsilon$ and putting $\epsilon=0$, we obtain

$$
\partial_{2} G\left(t, x_{1}(t), x_{2}(t)\right) \xi_{1}(t)+\partial_{3} G\left(t, x_{1}(t), x_{2}(t)\right) \xi_{2}(t)=0
$$

i.e.

$$
\partial_{2} G(t, x(t)) \xi_{1}(t)+\partial_{3} G(t, x(t)) \xi_{2}(t)=0 .
$$

Now, define the function

$$
\gamma(t)=-\frac{\partial_{3}\left(L_{\alpha, x}\right)-T_{\alpha}^{a}\left(\partial_{5}\left(L_{\alpha, x}\right)\right)}{\partial_{3} G(t, x(t))} .
$$

Using equations $(12)$ and $(13)$, we obtain

$$
\gamma(t) \partial_{2} G(t, x(t)) \xi_{1}(t)=\left[\partial_{3}\left(L_{\alpha, x}\right)-T_{\alpha}^{a}\left(\partial_{5}\left(L_{\alpha, x}\right)\right)\right] \xi_{2}(t) .
$$

On the other hand, using the fact that if $x$ is a minimizer of $\mathbb{J}$, then first variation of $\mathbb{J}$ is equal to zero, we have

$$
\begin{aligned}
& \int_{a}^{b}\left[\partial_{2}\left(L_{\alpha, x}\right) \xi_{1}(t)+\partial_{3}\left(L_{\alpha, x}\right) \xi_{2}(t)\right. \\
& \left.+\partial_{4}\left(L_{\alpha, x}\right) T_{\alpha}^{a} \xi_{1}(t)+\partial_{5}\left(L_{\alpha, x}\right) T_{\alpha}^{a} \xi_{2}(t)\right] d^{\alpha}(t, a)=0 .
\end{aligned}
$$

Using conformable integration by parts, we obtain

$$
\begin{aligned}
& \int_{a}^{b}\left[\left[\partial_{2}\left(L_{\alpha, x}\right)-T_{\alpha}^{a}\left(\partial_{4}\left(L_{\alpha, x}\right)\right)\right] \xi_{1}(t)\right. \\
& \left.+\left[\partial_{3}\left(L_{\alpha, x}\right)-T_{\alpha}^{a}\left(\partial_{5}\left(L_{\alpha, x}\right)\right)\right] \xi_{2}(t)\right] d^{\alpha}(t, a)=0 .
\end{aligned}
$$

Inserting (14) into the this integral, we get

$$
\int_{a}^{b}\left[\partial_{2}\left(L_{\alpha, x}\right)-T_{\alpha}^{a}\left(\partial_{4}\left(L_{\alpha, x}\right)\right)+\gamma(t) \partial_{2} G(t, x(t))\right] \xi_{1}(t) d^{\alpha}(t, a)=0 .
$$

Since $\xi_{1}$ is an arbitrary function, we can conclude that $x$ is a solution of

$$
\partial_{2}\left(L_{\alpha, x}\right)-T_{\alpha}^{a}\left(\partial_{4}\left(L_{\alpha, x}\right)\right)+\gamma(t) \partial_{2} G(t, x(t))=0 .
$$

Following the same process, the second condition

$$
\partial_{3}\left(L_{\alpha, x}\right)-T_{\alpha}^{a}\left(\partial_{5}\left(L_{\alpha, x}\right)\right)+\gamma(t) \partial_{3} G(t, x(t))=0
$$

can be obtained, and the proof is complete.

Theorem 9. Suppose that the function $L\left(t, x_{1}(t), x_{2}(t), y_{1}(t), y_{2}(t)\right)$ given by (8) is convex in $[a, b] \times \mathbb{R}^{4}, G \in \mathcal{C}_{2,3}^{1}$, and let $\gamma$ be given by equation [13). If $\partial_{3} G(t, x(t)) \neq 0$ for all $t \in[a, b]$ and $x$ is a solution of the fractional Euler-Lagrange equation (10), then $x$ minimizes $\mathbb{J}$ in $S^{*}$, subject to (9). 
Proof. If $x+\epsilon \xi$ is a variation of $x$, then we have

$$
\begin{aligned}
\mathbb{J}[x+\epsilon \xi]-\mathbb{J}[x] \geq & \int_{a}^{b}\left\{\left[\partial_{2}\left(L_{\alpha, x}\right)-T_{\alpha}^{a}\left(\partial_{4}\left(L_{\alpha, x}\right)\right)\right] \epsilon \xi_{1}(t)\right. \\
& +\left[\partial_{3}\left(L_{\alpha, x}\right)-T_{\alpha}^{a}\left(\partial_{5} L_{\alpha, x}\right)\right] \epsilon \xi_{2}(t) d^{\alpha}(t, a) .
\end{aligned}
$$

since the variation functions must satisfy the constraint $(9)$, from 12 we have the

$$
\xi_{2}(t)=-\frac{\partial_{2} G(t, x(t)) \xi_{1}(t)}{\partial_{3} G(t, x(t))}
$$

and from equation $(13)$, we obtain

$\mathbb{J}[x+\epsilon \xi]-\mathbb{J}[x] \geq \int_{a}^{b}\left[\partial_{2}\left(L_{\alpha, x}\right)-T_{\alpha}^{a}\left(\partial_{4}\left(L_{\alpha, x}\right)\right)+\gamma(t) \partial_{2} G(t, x(t))\right] \epsilon \xi_{1}(t) d^{\alpha}(t, a)$,

which is zero by hypothesis.

\section{Conclusions}

We have discussed the optimality conditions of the variational problems including conformable fractional derivatives. We obtained the optimality conditions for fixed end-point variational problems in Theorem 5 , and for variable end-point variational problems in Theorem 6 . Then, we have investigated the isoperimetric problem in Theorem 7, and variational problem with holonomic constraints in Theorem 8 . Finally, in Theorem 9, we have given a sufficient condition for optimality results of variational problems.

It is known that conformable fractional derivative generalizes the ordinary derivative, i.e. if we take $\alpha=1$ in conformable derivative $T_{\alpha}^{a} h(t)$, we have ordinary derivative $D h(t)$. Using this fact, It is clear that the results obtained in our study expand the results in the literature given before.

The problems we have dealt with include only one independent variable and one dependent variable and its derivative. As a possible extension of our results, one can study the problems involving more than one dependent variable and their derivatives. And problems with one dependent variable and its derivatives of different orders can be studied.

Authors Contribution Statement All authors contributed equally and significantly in this manuscript, and they read and approved the final manuscript.

Declaration of Competing Interests The authors declare that they have no competing interests. 


\section{REFERENCES}

[1] Abdeljawad, T., On conformable fractional calculus, J. Comput. Appl. Math., 279 (2015), 57-66. https://doi.org/10.1016/j.cam.2014.10.016

[2] Agarwal, O. P., Formulation of Euler-Lagrange equations for fractional variational problems, J. Math. Anal. Appl., 272 (2002), 368-379. https://doi.org/10.1016/S0022-247X(02)00180-4

[3] Agarwal, O. P., Fractional variational calculus and the transversality conditions, J. Phys. A, 39(33) (2006), 10375-10384. https://doi.org/10.1088/0305-4470/39/33/008

[4] Agarwal, O. P., Fractional variational calculus in terms of Riesz fractional derivatives, J. Phys. A, 40(24) (2007), 6287-6303. https://doi.org/10.1088/1751-8113/40/24/003

[5] Almeida, R., Fractional variational problems with the Riesz-Caputo derivative, Appl. Math. Lett., 25(2) (2012), 142-148. https://doi.org/10.1016/j.aml.2011.08.003

[6] Almeida, R., Variational problems involving a Caputo-type fractional derivative, J. Optim. Theory Appl., 174(1) (2017), 276-294. https://doi.org/10.1007/s10957-016-0883-4

[7] Bastos, N. R. O., Calculus of variations involving Caputo-Fabrizio fractional differentiation, Stat., Optim. Inf. Comput., 6 (2018), 12-21. https://doi.org/10.19139/soic.v6i1.466

[8] Batarfi, H., Losada, J., Nieto, J. J., Shammakh, W., Three-point boundary value problems for conformable fractional differential equations, J. Funct. Spaces, 2015, Art. ID 706383, 6 pp. https://doi.org/10.1155/2015/706383

[9] Chatibi, Y., El Kinani, E. H., Ouhadan, A., Lie symmetry analysis of conformable differential equations, AIMS Math., 4(4) (2019), 1133-1144. https://doi.org/10.3934/math.2019.4.1133

[10] Chatibi, Y., El Kinani, E. H., Ouhadan, A., Variational calculus involving nonlocal fractional derivative with Mittag-Leffler kernel, Chaos, Solitons $\mathcal{E}$ Fractals, 118 (2019), 117-121. https://doi.org/10.1016/j.chaos.2018.11.017

[11] Chatibi, Y., El Kinani, E. H., Ouhadan, A., Lie symmetry analysis and conservation laws for the time fractional Black-Scholes equation, International Journal of Geometric Methods in Modern Physics, 17(01) (2020), 2050010. https://doi.org/10.1142/S0219887820500103

[12] Chatibi, Y., El Kinani, E. H., Ouhadan, A., On the discrete symmetry analysis of some classical and fractional differential equations, Math. Methods Appl. Sci., 44(4) (2021), 28682878. https://doi.org/10.1002/mma.6064

[13] Chung, W. S., Fractional Newton mechanics with conformable fractional derivative, J. Comput. Appl. Math., 290 (2015), 150-158. https://doi.org/10.1016/j.cam.2015.04.049

[14] Çenesiz, Y., Kurt, A., The solutions of time and space conformable fractional heat equations with conformable Fourier transform, Acta Univ. Sapientiae Math., 7(2) (2015), 130-140. https://doi.org/10.1515/ausm-2015-0009

[15] Diethelm, K., The Analysis of Fractional Differential Equations, Springer, 2010.

[16] Eroğlu, B. B. I., Yapışkan, D., Generalized conformable variational calculus and optimal control problems with variable terminal conditions, AIMS Mathematics, 5 (2020), 1105-1126. https://doi.org/10.3934/math.2020077

[17] Gelfand, I. M., Fomin, S. V., Calculus of variations, Prentice-Hall, Inc., 1963.

[18] Kadkhoda, N., Jafari, H., An analytical approach to obtain exact solutions of some space-time conformable fractional differential equations, Adv. Difference Equ., 2019, Paper No. 428, 10 pp. https://doi.org/10.1186/s13662-019-2349-0

[19] Khalil, R., Al Horani, M., Yousef, A., Sababheh, M., A new definition of fractional derivative, J. Comput. Appl. Math., 264 (2014), 65-70. https://doi.org/10.1016/j.cam.2014.01.002

[20] Kilbas, A. A., Srivastava, H. M., Trujillo, J. J., Theory and Applications of Fractional Differential Equations, Elsevier Science, 2006.

[21] Lazo, M. J., Torres, D. F. M., Variational calculus with conformable fractional derivatives, IEEE/CAA Journal of Automatica Sinica, 4(2) (2017), 340-352. https://doi.org/10.1109/JAS.2016.7510160 
[22] Machado, J. T., Kiryakova, V., Mainardi, F., Recent history of fractional calculus, Commun. Nonlinear Sci. Numer. Simul., 16(3) (2011), 1140-1153. https://doi.org/10.1016/j.cnsns.2010.05.027

[23] Mainardi, F., An historical perspective on fractional calculus in linear viscoelasticity, Fract. Calc. Appl. Anal., 15(4) (2012), 712-717. https://doi.org/10.2478/s13540-012-0048-6

[24] Miller, K. S., Ross, B., An Introduction to Fractional Calculus and Fractional Differential Equations, Wiley Interscience, 1993.

[25] Riewe, F., Nonconservative Lagrangian and Hamiltonian mechanics, Phys. Rev. E, 53(2) (1996), 1890-1899. https://doi.org/10.1103/PhysRevE.53.1890

[26] Riewe, F., Mechanics with fractional derivatives, Phys. Rev. E, 55(3) (1997), 3581-3592. https://doi.org/10.1103/PhysRevE.55.3581

[27] Ross., B., A brief history and exposition of the fundamental theory of fractional calculus, pages 1-36, Springer Berlin Heidelberg, Berlin, Heidelberg, 1975.

[28] Weberszpil, J., Helayel-Neto, J. A., Variational approach and deformed derivatives, Physica A, 450 (2016), 217-227. https://doi.org/10.1016/j.physa.2015.12.145

[29] Zhang, J., Ma, X., Li, L., Optimality conditions for fractional variational problems with Caputo-Fabrizio fractional derivatives, Adv. Differ. Equ., 357 (2017). https://doi.org/10.1186/s13662-017-1388-7 